\title{
Large-Scale Cohort Study on the Relationship Between Serum Lipid Concentrations and Risk of Cerebrovascular Disease Under Low-Dose Simvastatin in Japanese Patients With Hypercholesterolemia
}

\section{Sub-Analysis of the Japan Lipid Intervention Trial (J-LIT)}

\author{
Noriaki Nakaya, MD; Toru Kita, MD*; Hiroshi Mabuchi, MD**; Masunori Matsuzaki, MD ${ }^{\dagger}$; \\ Yuji Matsuzawa, MD ${ }^{\dagger}$; Shinichi Oikawa, MD $\$$; Yasushi Saito, MD $\$$; \\ Jun Sasaki, MD\$; Kazuaki Shimamoto, MD\$\$; \\ Hiroshige Itakura, MDI, III; the J-LIT Study Group
}

\begin{abstract}
Background The Japan Lipid Intervention Trial was a nationwide cohort study of 52,421 hypercholesterolemic patients treated with open-labeled simvastatin for 6 years under standard clinical practices. Cerebrovascular disease (CVD) is one of the leading causes of death in Japan, but the effect of hypercholesterolemia on CVD has not been well established in Japanese patients. This study aimed to determine the relationship between the risk of CVD and serum lipid concentrations during treatment in Japan.

Methods and Results Patients were treated with 5-10 mg/day of simvastatin and all, including those who discontinued simvastatin for any reason, had their lipid concentrations and incidence of CVD monitored for 6 years. Data of 41,088 patients were analyzed in this study, excluding those who had a history of coronary heart disease or CVD. The risk of cerebral infarction was higher in patients whose mean total cholesterol concentrations during treatment were $\geq 240 \mathrm{mg} / \mathrm{dl}$, low-density lipoprotein cholesterol concentrations $\geq 160 \mathrm{mg} / \mathrm{dl}$, triglycerides $\geq 150 \mathrm{mg} / \mathrm{dl}$ and high-density lipoprotein cholesterol concentrations $<40 \mathrm{mg} / \mathrm{dl}$. There was no obvious correlation between cerebral hemorrhage and serum lipid concentrations.

Conclusion Improvement of serum lipid concentrations is important for reducing the incidence of cerebral infarction. (Circ J 2005; 69: 1016-1021)
\end{abstract}

Key Words: Cerebrovascular disease; Lipids; Primary prevention

C erebrovascular disease (CVD) is one of the major causes of death in Japan ${ }^{1}$ and is a leading cause of disability and increased healthcare costs. A number of studies have already demonstrated that lipid-lowering therapy reduces the risk of coronary heart disease (CHD), but its effect on CVD has not been well established in Japan. The Kyushu Lipid Intervention Study demonstrated a correlation between the serum lipid concentration and the incidence of cerebral infarction, but the number of patients was relatively small, ${ }^{2,3}$ Several epidemiologic studies have failed to demonstrate an association between cholesterol concentrations and $\mathrm{CVD}_{4}^{4-7}$ although a post hoc finding in the Scandinavian Simvastatin Survival Study

(Received January 5, 2005; revised manuscript received May 16, 2005; accepted June 8, 2005)

Nakaya Clinic, Tokyo, *Kyoto University Graduate School of Medicine, Kyoto, **Kanazawa University Graduate School of Medicine, Kanazawa, Yamaguchi University Graduate School of Medicine, Ube, †Sumitomo Hospital, Osaka, $\$$ Nippon Medical School, Tokyo, \#Chiba University Graduate School of Medicine, Chiba, \$nternational University of Health and Welfare Graduate School, Fukuoka, $\$$ Sapporo Medical University School of Medicine, Sapporo, IIbaraki Christian University, Hitachi, and IIIChairman of Central Committee, Tokyo, Japan

Mailing address: Noriaki Nakaya, MD, Nakaya Clinic, 4-7-8 Higashinakano, Nakano-ku, Tokyo 164-0003, Japan
(4S) showed that simvastatin reduced the incidence of CVD by $30 \% 8$ A pooled analysis of 4 trials conducted primarily in CHD patients showed a $62 \%$ lower rate of total CVD attributable to pravastatin? The Heart Protection Study reported that simvastatin reduced the incidence rate of total and ischemic CVD in UK adults with high risk ${ }^{10}$ by 25\% and 30\%, respectively, and the Anglo-Scandinavian Cardiac Outcomes Trial study reported that atorvastatin reduced the incidence of CVD in hypertensive patients by 27\%:11 In Japan, the Oyabe Study reported that the incidence of CVD in Japanese subjects with low concentrations of high-density lipoprotein cholesterol (HDL-C $<30 \mathrm{mg} / \mathrm{dl}$ ) was remarkably higher than that in subjects with high HDL-C $(\geq 60 \mathrm{mg} / \mathrm{dl})$ and the incidence of CVD was higher in the subjects with total cholesterol (TC) concentrations over $220 \mathrm{mg} / \mathrm{dl} !^{12}$ The Eastern Stroke and Coronary Heart Disease Collaborative Research Group reported that there was a trend toward a decrease in the risk of ischemic CVD and an increase in the risk of hemorrhagic CVD with decreasing cholesterol concentrations in populations from eastern Asia! ${ }^{3}$

The Japan Lipid Intervention Trial (J-LIT) was a nationwide cohort study of 52,421 hypercholesterolemic patients treated with open-label simvastatin $(5-10 \mathrm{mg} /$ day $)$, carried out for 6 years by a large number of physicians under standard clinical practices in order to evaluate the relationship 
between cardiovascular disease and lipid concentrations ${ }^{14-16}$ The results clearly showed that normalization of the lipid concentration of hypercholesterolemic patients reduced the risk of coronary events. In the present study, we analyzed the relationship between the risk of CVD and lipid concentrations.

\section{Methods}

\section{Subjects}

The J-LIT study enrolled 52,421 patients with serum TC concentration $\geq 220 \mathrm{mg} / \mathrm{dl}$ : men aged $35-70$ years and postmenopausal women under 70 years old. Patients who had been treated with a lipid-lowering agent were screened for eligibility after a washout period of at least 4 weeks; the washout period was at least 12 weeks for patients previously treated with probucol. Exclusion criteria included recent acute myocardial infarction or CVD, uncontrolled diabetes mellitus, serious concomitant hepatic or renal disease, secondary hypercholesterolemia, malignancy or any other illness with a poor prognosis. For this study, 41,088 patients without a history of CHD or CVD were selected.

\section{Study Design}

The design of the J-LIT study has been described previously! ${ }^{17}$ Patients were treated with open-label simvastatin according to the approved Japanese labeling of Lipovas ${ }^{\circledR}$. All patients, including those who discontinued simvastatin for any reason, were monitored for 6 years. Their lipid concentrations, adverse events, and incidence of CHD-related events and CVD were recorded. Cholesterol concentrations were determined locally at the study institutions. Dietary and exercise therapies for hyperlipidemia were recommended to patients by the investigators and additional lipid-lowering agents were allowed at the discretion of the physician. No restrictions were placed on the administration of medical treatment for complications. The low-density lipoprotein cholesterol (LDL-C) concentration in patients with triglyceride (TG) concentration $\leq 400 \mathrm{mg} / \mathrm{dl}$ was calculated using the Friedewald formula ${ }^{18}$ Body weight, blood pressure, and the serum lipid concentrations were measured every 6 months after enrollment and patients were asked about drug compliance, number of cigarettes smoked, alcohol consumption, and amount of exercise. Every 12 months, hepatic and renal functions were monitored, and an ECG was recorded.

The type of CVD was determined using clinical symptoms, angiography and/or computed tomography (CT), and classified according to the criteria of the Stroke Committee established by the Japanese Ministry of Education. 19 The pre-specified primary endpoints of the sub-analysis were cerebral infarction, including cerebral thrombosis and cerebral embolism, and cerebral hemorrhage pre-specified in the protocol. The first event or death that occurred during the study period were counted once in each patient, and the events were reviewed and determined by the Endpoint Classification Committee. CVD events were counted once. The follow-up data acquired after the onset of disease other than the endpoints were excluded from the analysis. Each patient was informed of the study purpose, as well as drug efficacy and need for long-term treatment. Written informed consent was not obtained from patients, because commercially available simvastatin preparation was used for the open-label study.
Table 1 Baseline Characteristics and Lipid Profiles of the Patients

\begin{tabular}{|c|c|}
\hline$N$ & 41,088 \\
\hline Males (\%) & 31.6 \\
\hline Age (years) & $57.7 \pm 7.9$ \\
\hline Body mass index $\left(\mathrm{kg} / \mathrm{m}^{2}\right)$ & $24.0 \pm 3.2$ \\
\hline Hypertension (\%) & 45.6 \\
\hline Diabetes mellitus (\%) & 15.1 \\
\hline ECG abnormal (\%) & 12.8 \\
\hline Coronary heart disease familial history (\%) & 4.7 \\
\hline Smoking habit (\%) & 16.5 \\
\hline Alcohol consumption (\%) & 29 \\
\hline \multicolumn{2}{|l|}{ Baseline } \\
\hline Total cholesterol $(\mathrm{mg} / \mathrm{dl})$ & $270 \pm 34$ \\
\hline Low-density lipoprotein cholesterol ( $\mathrm{mg} / \mathrm{dl})$ & $182 \pm 33$ \\
\hline Triglyceride $(m g / d l)$ & $196 \pm 171$ \\
\hline High-density lipoprotein cholesterol ( $\mathrm{mg} / \mathrm{dl})$ & $53 \pm 15$ \\
\hline \multicolumn{2}{|l|}{ During treatment } \\
\hline Total cholesterol $(\mathrm{mg} / \mathrm{dl})$ & $220 \pm 30$ \\
\hline Low-density lipoprotein cholesterol ( $\mathrm{mg} / \mathrm{dl})$ & $134 \pm 30$ \\
\hline Triglyceride $(\mathrm{mg} / \mathrm{dl})$ & $164 \pm 103$ \\
\hline High-density lipoprotein cholesterol $(\mathrm{mg} / \mathrm{dl})$ & $55 \pm 14$ \\
\hline
\end{tabular}

\section{Statistical Analysis}

All data, including those obtained after the termination of simvastatin therapy, were analyzed by survival analysis. The mean lipid concentrations were calculated using the data obtained throughout the treatment period, because the mean lipid concentrations were thought to be important for clinical practice. The data for lipid concentrations acquired after the onset of disease were excluded. For analysis of baseline patient age and lipid profile, continuous variables within and between subgroups were assessed using the paired or unpaired t-test. Patients were classified into 3-6 subgroups based on the mean lipid concentrations during the treatment. TC, TG, LDL-C, and HDL-C concentrations and the ratio of LDL-C/HDL-C were classified into discrete intervals of $20,150,20$ and $10 \mathrm{mg} / \mathrm{dl}$ and 0.5 , respectively. The reference categories were set on the subgroups with the lowest concentrations.

We calculated the relative risks, with $95 \%$ confidence intervals for each endpoint, of each subgroup relative to the reference category, using the Cox proportional-hazards model with adjustment for gender and age at baseline (as a continuous variable), hypertension, diabetes mellitus, and smoking habit. The effects of each baseline characteristic on each endpoint were assessed, except for the effect of age, which was categorized. Data are expressed as the mean \pm SD. For all statistical analyses, a p-value $<0.05$ was considered to be significant. All statistical calculations were performed using SAS software (version 6.12, SAS Institute Inc, Cary, NC, USA).

\section{Results}

\section{Follow-up}

A total of 42,360 of the 52,421 patients enrolled in the J-LIT study were eligible for the primary prevention analysis of CHD and their clinical characteristics have been reported previously! ${ }^{17}$ In the present study, data were collected from 41,088 patients and 1,272 patients were excluded on the history of CVD. In total, 30,832 patients were followed up by the investigators to the end of the 6th year (mean follow-up, 5.39 years per subject). Baseline characteristics are shown in Table 1. Approximately $46 \%$ of patients had hypertension (mean systolic and diastolic 
Table 2 Incidence of Cerebrovascular Events During Follow-up Period

\begin{tabular}{|c|c|c|c|c|c|c|}
\hline & \multicolumn{2}{|c|}{ Fatal } & \multicolumn{2}{|c|}{ Non-fatal } & \multicolumn{2}{|c|}{ Total } \\
\hline & No. of events & Incidence & No. of events & Incidence & No. of events & Incidence \\
\hline Cerebral infarction & 22 & $(0.10)$ & 220 & $(1.00)$ & 242 & (1.09) \\
\hline Cerebral thrombosis & 12 & $(0.05)$ & 139 & $(0.63)$ & 151 & $(0.68)$ \\
\hline Cerebral embolism & 10 & $(0.05)$ & 81 & $(0.37)$ & 91 & $(0.41)$ \\
\hline Cerebral hemorrhage* & 22 & $(0.10)$ & 80 & $(0.36)$ & 102 & $(0.46)$ \\
\hline
\end{tabular}

*Intracranial. Incidence =/1,000 patients-year.

Table 3 Relative Risk of Cerebrovascular Disease and Serum Lipid Concentrations During Treatment

\begin{tabular}{|c|c|c|c|c|c|c|c|c|c|}
\hline & \multicolumn{5}{|c|}{ Cerebral infarction } & \multicolumn{4}{|c|}{ Cerebral hemorrhage* } \\
\hline & Population & Event & Relative risk & $95 \% C I$ & $p$ value & Event & Relative risk & $95 \% C I$ & $p$ value \\
\hline \multicolumn{10}{|l|}{$T C(m g / d l)$} \\
\hline$<200$ & 9,306 & 50 & 1.00 & & & 29 & 1.00 & & \\
\hline $200-219$ & 12,119 & 70 & 1.26 & $(0.87-1.81)$ & 0.216 & 34 & 0.98 & $(0.60-1.61)$ & 0.940 \\
\hline $220-239$ & 10,423 & 59 & 1.38 & $(0.94-2.01)$ & 0.100 & 17 & 0.61 & $(0.34-1.12)$ & 0.112 \\
\hline $240-259$ & 5,275 & 34 & 1.76 & $(1.13-2.73)$ & 0.012 & 11 & 0.85 & $(0.42-1.71)$ & 0.638 \\
\hline$\geq 260$ & 3,423 & 28 & 2.49 & $(1.56-3.99)$ & $<0.001$ & 9 & 1.15 & $(0.54-2.46)$ & 0.718 \\
\hline \multicolumn{10}{|c|}{$L D L-C(m g / d l)$} \\
\hline$<120$ & 12,860 & 74 & 1.00 & & & 32 & 1.00 & & \\
\hline $120-139$ & 12,260 & 76 & 1.23 & $(0.89-1.70)$ & 0.200 & 39 & 1.41 & $(0.88-2.26)$ & 0.151 \\
\hline $140-159$ & 8,871 & 36 & 0.89 & $(0.60-1.33)$ & 0.575 & 15 & 0.82 & $(0.44-1.51)$ & 0.515 \\
\hline $160-179$ & 3,853 & 30 & 1.92 & $(1.25-2.94)$ & 0.003 & 7 & 0.95 & $(0.42-2.16)$ & 0.902 \\
\hline$\geq 180$ & 2,318 & 24 & 2.95 & $(1.85-4.71)$ & $<0.001$ & 6 & 1.51 & $(0.62-3.63)$ & 0.363 \\
\hline \multicolumn{10}{|l|}{$T G(m g / d l)$} \\
\hline$<150$ & 22,413 & 101 & 1.00 & & & 53 & 1.00 & & \\
\hline $150-299$ & 15,577 & 115 & 1.54 & $(1.18-2.02)$ & 0.002 & 41 & 0.99 & $(0.65-1.50)$ & 0.957 \\
\hline$\geq 300$ & 2,532 & 25 & 2.31 & $(1.47-3.63)$ & $<0.001$ & 5 & 0.73 & $(0.28-1.86)$ & 0.504 \\
\hline \multicolumn{10}{|c|}{$H D L-C(m g / d l)$} \\
\hline$<40$ & 4,001 & 48 & 1.00 & & & 16 & 1.00 & & \\
\hline $40-49$ & 11,522 & 70 & 0.52 & $(0.36-0.75)$ & $<0.001$ & 31 & 0.68 & $(0.37-1.25)$ & 0.217 \\
\hline $50-59$ & 12,162 & 66 & 0.49 & $(0.33-0.71)$ & $<0.001$ & 25 & 0.54 & $(0.29-1.03)$ & 0.061 \\
\hline$\geq 60$ & 12,861 & 57 & 0.41 & $(0.28-0.61)$ & $<0.001$ & 28 & 0.60 & $(0.32-1.13)$ & 0.117 \\
\hline \multicolumn{10}{|c|}{$L D L-C / H D L-C$} \\
\hline$<2.0$ & 10,439 & 55 & 1.00 & & & 25 & 1.00 & & \\
\hline $2.0-2.4$ & 9,904 & 46 & 0.90 & $(0.61-1.33)$ & 0.597 & 24 & 1.02 & $(0.58-1.79)$ & 0.943 \\
\hline $2.5-2.9$ & 8,682 & 48 & 1.11 & $(0.75-1.63)$ & 0.611 & 17 & 0.84 & $(0.45-1.56)$ & 0.582 \\
\hline $3.0-3.4$ & 5,578 & 37 & 1.37 & $(0.91-2.09)$ & 0.136 & 12 & 0.95 & $(0.48-1.89)$ & 0.877 \\
\hline$\geq 3.5$ & 5,559 & 54 & 2.13 & $(1.46-3.11)$ & $<0.001$ & 21 & 1.75 & $(0.97-3.14)$ & 0.061 \\
\hline Total & 40,546 & 241 & & & & 100 & & & \\
\hline
\end{tabular}

Cerebral infarction, cerebral thrombosis and cerebral embolism; *Intracranial.

CI, confidence interval; TC, total cholesterol; LDL-C, low-density lipoprotein cholesterol; TG, triglyceride; HDL-C, high-density lipoprotein cholesterol.

blood pressures 150 and $87 \mathrm{mmHg}$, respectively), and $15 \%$ had diabetes mellitus. Hypertension was treated with angiotensin-converting enzyme inhibitors $(26 \%)$, calciumchannel blockers $(53 \%), \beta$-blockers $(18 \%)$ and diuretics $(6 \%)$.

\section{Changes in Serum Lipid Concentrations With Simvastatin Treatment}

The mean serum concentrations of TC, LDL-C, and TG at baseline were $270 \pm 34,182 \pm 33$, and $196 \pm 171 \mathrm{mg} / \mathrm{dl}$, respectively, and the mean lipid concentrations during treatment were $220 \pm 30 \mathrm{mg} / \mathrm{dl}, 134 \pm 30 \mathrm{mg} / \mathrm{dl}$ and $164 \pm$ $103 \mathrm{mg} / \mathrm{dl}$, respectively. The mean serum HDL-C concentration increased from baseline of $53 \pm 15 \mathrm{mg} / \mathrm{dl}$ to $55 \pm$ $14 \mathrm{mg} / \mathrm{dl}$. The mean percent change from baseline in the TC, LDL-C, TG, and HDL-C concentrations was $-18 \%$, $-27 \%,-16 \%$, and $+4 \%$, respectively, during the treatment period.

Incidence of CVD During the Treatment Period

Cerebral infarction occurred in 242 patients during the course of the study with an incidence of 1.09 events per 1,000 patients-year (Table 2). Cerebral thrombosis occurred in 151 patients, and cerebral embolism in 91 patients. Cerebral hemorrhage occurred in 102 patients with an incidence of 0.46 events per 1,000 patients-year. The incidence of total CVD was 1.55 events per 1,000 patients-year. CT of the head was performed in 77\% of the patients with CVD.

\section{Relationship Between the Relative Risk of Cerebral Infarction and Lipid Concentrations}

The relative risk of cerebral infarction was higher in patients with TC concentration $\geq 240 \mathrm{mg} / \mathrm{dl}$ compared to those with TC concentration $<200 \mathrm{mg} / \mathrm{dl}$ (Table 3 ) and patients with LDL-C concentration $\geq 160 \mathrm{mg} / \mathrm{dl}$ had higher risk than those with a concentration $<120 \mathrm{mg} / \mathrm{dl}$. A group of patients with a mean TG concentration $\geq 150 \mathrm{mg} / \mathrm{dl}$ had a higher incidence of cerebral infarction than those with a mean concentration $<150 \mathrm{mg} / \mathrm{dl}$. The incidence of cerebral infarction was lower in patients with a mean HDL-C concentration of $40-49 \mathrm{mg} / \mathrm{dl}$ compared with a concentration $<40 \mathrm{mg} / \mathrm{dl}$. The relative risk of cerebral infarction was 

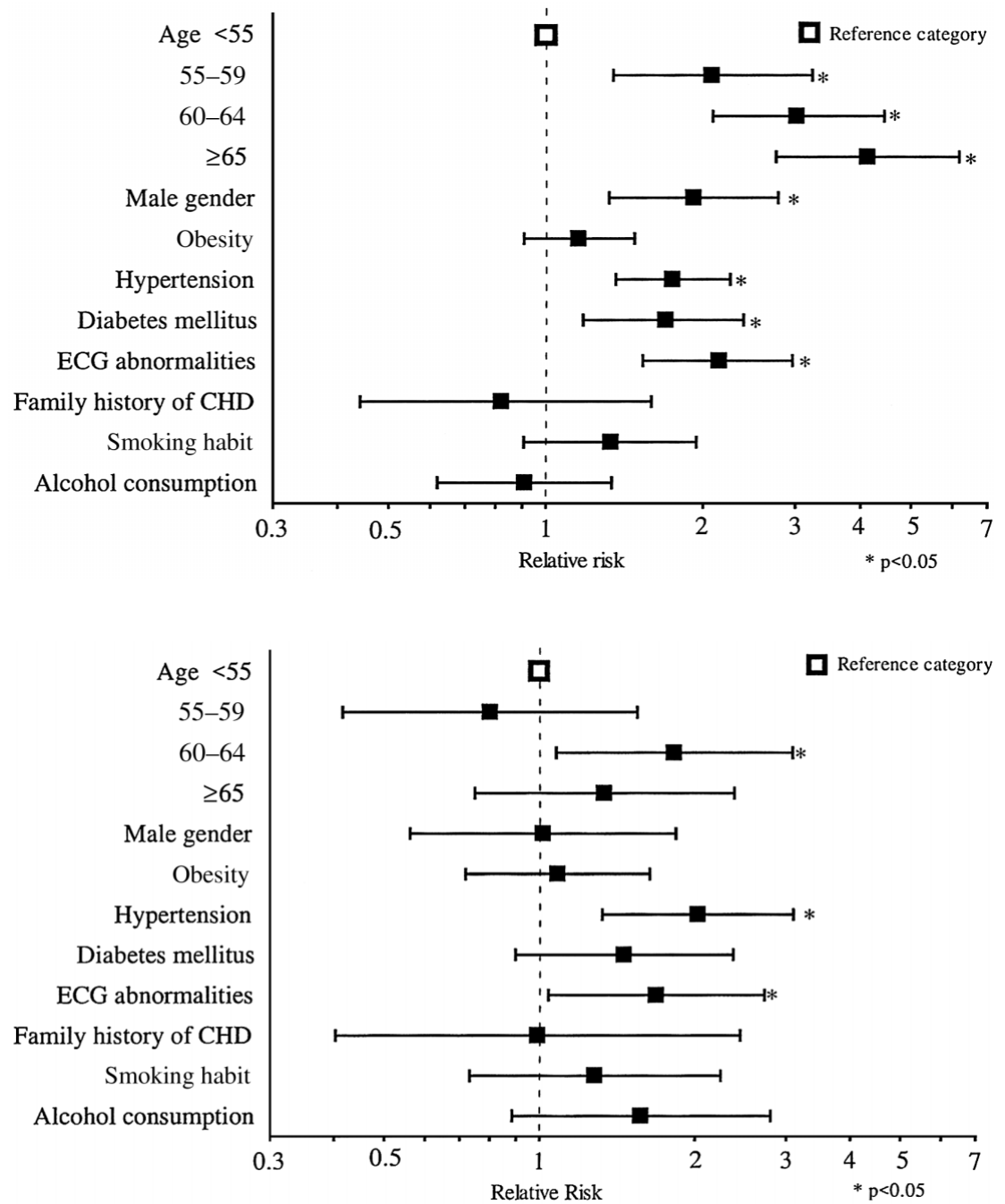

Fig 1. Relationship between the relative risk of cerebral infarction and baseline characteristics of patients maintained on low-dose simvastatin. Data were adjusted for age, sex, hypertension, diabetes mellitus and smoking habit. Bars express the relative risk with a $95 \%$ confidence interval. Obesity = body mass index $(\mathrm{BMI}) \geq 25 \mathrm{~kg} / \mathrm{m}^{2}$. CHD, coronary heart disease.
Fig 2. Relationship between the relative risk of cerebral hemorrhage and baseline characteristics of patients maintained on low-dose simvastatin. Data were adjusted for age, sex, hypertension, diabetes mellitus and smoking habit. Bars express the relative risk with a $95 \%$ confidence interval. Obesity $=$ body mass index $(\mathrm{BMI}) \geq 25 \mathrm{~kg} / \mathrm{m}^{2}$. CHD, coronary heart disease. higher in patients with the ratio of LDL-C/HDL-C $\geq 3.5$ compared with a ratio $<2.0$.

\section{Relationship Between the Relative Risk of Cerebral Infarction and Baseline Characteristics}

The risk of cerebral infarction was analyzed using multiple regression (Fig 1). Age correlated with the incidence of cerebral infarction and male patients had higher risk compared with female patients. Hypertension, diabetes mellitus, and ECG abnormalities were also risk factors for cerebral infarction. Alcohol consumption, cigarette smoking and a family history of CHD were not risk factors.

\section{Relationship Between the Relative Risk of Cerebral Hemorrhage and Mean Lipid Concentrations}

There was no clear relationship between cerebral hemorrhage and the concentrations of TC, LDL-C, TG and HDL$\mathrm{C}$ or the ratio of LDL-C/HDL-C (Table 3 ).

\section{Relationship Between the Relative Risk of Cerebral Hemorrhage and Baseline Characteristics}

The risk of cerebral hemorrhage was analyzed by multiple regression (Fig 2), which showed that patients aged 60-64 years had a significantly higher risk of cerebral hemorrhage. Hypertension and ECG abnormalities were also risk factors for cerebral hemorrhage.

\section{Relationship Between the Relative Risk of Cerebral} Infarction and Hypertension

Hypertension is known to be a dominant risk factor for cerebral infarction, so the effect of lipid concentrations on cerebral infarction was examined in patients stratified by systolic blood pressure (SBP). Table 4 shows the relationship between cerebral infarction and serum lipid concentrations, comparing a SBP at baseline $<140 \mathrm{mmHg}$ and SBP $\geq 140 \mathrm{mmHg}$. The influence of serum lipid concentrations on cerebral infarction was observed in both the hypertensive and normotensive groups and was also observed in patients stratified by diastolic blood pressure $(<90 \mathrm{mmHg}$ and $\geq 90 \mathrm{mmHg}$; data not shown). The serum lipid concentrations are thus independent risk factors of cerebral infarction.

\section{Discussion}

We analyzed the relationship between the risk of CVD and lipid concentrations using the J-LIT data. During the 6-year treatment period simvastatin reduced the serum concentrations of TC, LDL-C and TG by $18 \%, 27 \%$ and $16 \%$, respectively, from baseline. The concentration of HDL-C continued to increase during the treatment period. The incidence of CVD in Japanese patients without prior CVD or CHD was 1.55 events per 1,000 patients-year in this study, much higher than for primary CHD ( 0.91 events 
Table 4 Relative Risk of Cerebral Infarction and Serum Lipid Concentrations During Treatment

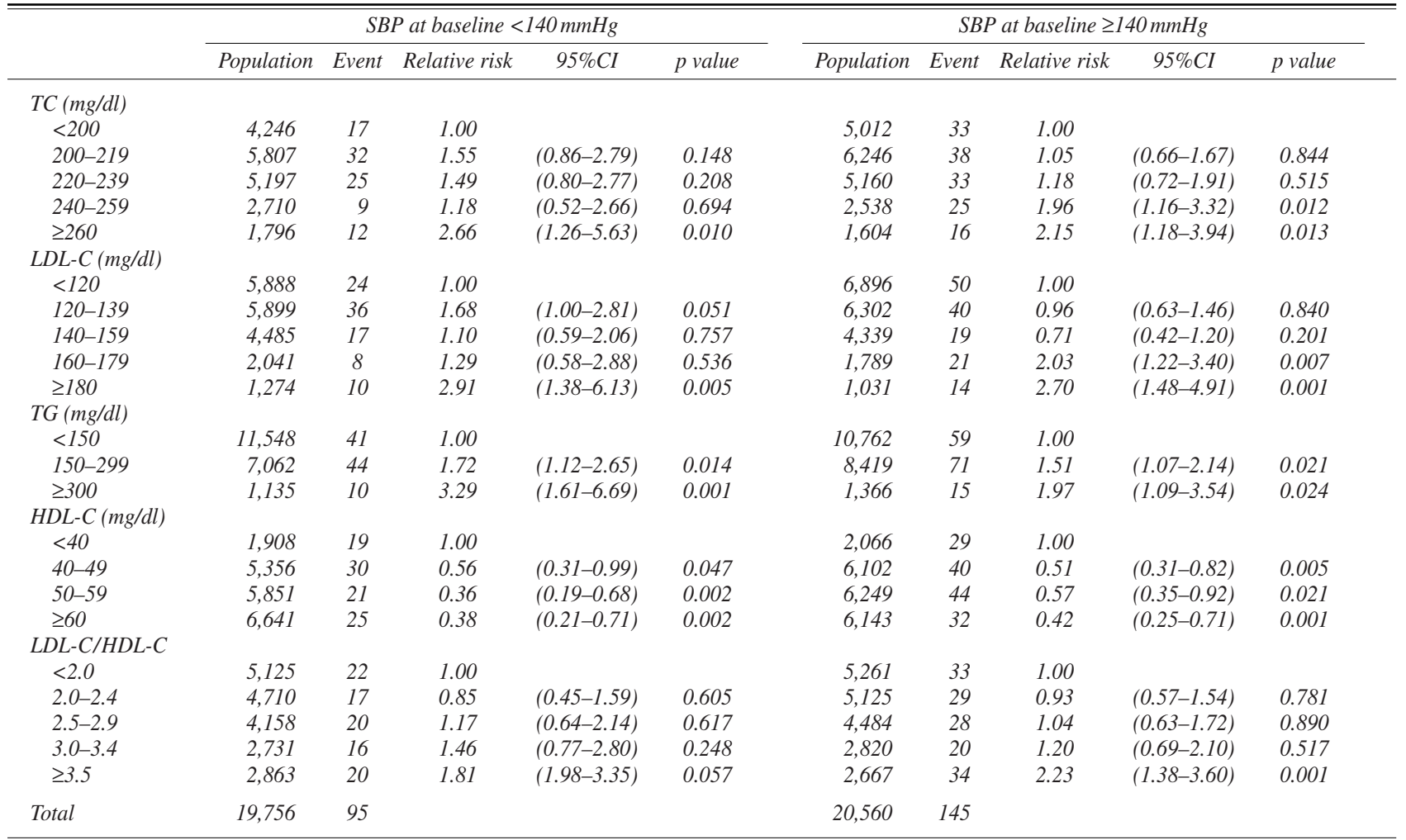

Cerebral infarction, cerebral thrombosis and cerebral embolism.

SBP, systolic blood pressure. Other abbreviations see Table 3.

per 1,000 patients-year ${ }^{14}$ ), and indicates that in Japan the prevention of CVD is as important as that of CHD. The risk of cerebral infarction was higher when the mean TC concentration was $\geq 240 \mathrm{mg} / \mathrm{dl}$ and the mean LDL-C concentration $\geq 160 \mathrm{mg} / \mathrm{dl}$, which are the same values for the risk of CHD. The incidence of cerebral infarction increased in the patients with TG concentration $\geq 150 \mathrm{mg} / \mathrm{dl}$, whereas the incidence of CHD increased when TG concentration was $\geq 300 \mathrm{mg} / \mathrm{dl}$, which indicates a closer relationship between cerebral infarction and TG than between CHD and TG. HDL-C inversely correlated with the risk of cerebral infarction. There was no obvious correlation between cerebral hemorrhage and serum lipid concentrations nor could we observe a clear relationship between the incidence of fatal CVD and lipid concentrations. In the preliminary analysis, no different results were obtained when adjusted for SBP. In a meta-analysis of randomized trials, 20 lipidlowering therapy reduced the overall CVD incidence, but not that of fatal CVD incidence and it was concluded that the incidence of hemorrhagic CVD was not influenced by lipid-lowering therapy. Because hemorrhagic CVD is mostly fatal could partly explain the apparent lack of effect of lipid-lowering therapy on fatal CVD in that analysis and might be applicable to our results.

Our findings are generally consistent with the results from the Oyabe Study, a community-based 10-year followup study of Japanese men and women, 12 although the incidence was much less (crude rates for all CVD incidence per 1,000 person-years were 4.30 in men and 1.98 in women in the Oyabe study). Low HDL-C concentrations $(<30 \mathrm{mg} / \mathrm{dl})$ led to a significantly and independently increased risk of CVD. The incidence of CVD was higher in the subjects with TC concentrations $>220 \mathrm{mg} / \mathrm{dl}$ and the relationship of TC to the overall incidence of CVD and that of ischemic CVD in particular showed a U-shaped curve with the bottom between TC values of 180 and $220 \mathrm{mg} / \mathrm{dl}$. In the present J-LIT study, the incidence of ischemic CVD tended to increase in patients with TC concentrations $<180 \mathrm{mg} / \mathrm{dl}$, but was not significant (data not shown). The patients who participated in this study were all treated with low-dose of simvastatin, but our results are similar to the result of the Oyabe Study. The Eastern Stroke and Coronary Heart Disease Collaborative Research Group ${ }^{13}$ reported that there were trends towards a decrease in the risk of nonhemorrhagic CVD and an increase in the risk of hemorrhagic CVD with decreasing cholesterol concentrations, and in the present study, high TC, LDL-C and TG concentrations and low HDL-C concentrations were risk factors for cerebral infarction. The influence of serum lipid concentrations on cerebral infarction (thrombosis and embolism) was similar in males and females. In the analysis of patients with TC concentrations $<180 \mathrm{mg} / \mathrm{dl}$, the incidence of cerebral hemorrhage was not increased, compared with those with a TC concentration $\geq 180 \mathrm{mg} / \mathrm{dl}$. A relationship between the effect of lipid concentrations and cerebral infarction, analyzed by hypertension, sex, and types of cerebral infarction, was clearly observed in this study.

The antiatherothrombotic properties of statins, ${ }^{21}$ apart from their cholesterol-lowering effects, may be the mechanism of the reduction in cardiovascular events. The thrombotic sequelae of plaque disruption may be mitigated by statins through their inhibition of platelet aggregation and maintenance of a favorable balance between prothrombic and fibrinolytic mechanisms. ${ }^{21}$ These same antiatherothrom- 
botic effects may have also affected the present results.

This J-LIT subanalysis is the first report of a correlation between the incidence of cerebral infarction and lipid concentrations in a large number of Japanese hypercholesterolemic patients. Our results show that improving the serum lipid concentrations is also important for reducing the incidence of cerebral infarction.

\section{Study Limitation}

Although we refer to this study as J-LIT, in reality it was a cohort and observational study rather than an interventional study.

\section{Acknowledgment}

This study was in part supported by a grant from Banyu Pharmaceutical Co, Ltd, Tokyo, Japan.

\section{References}

1. Ministry of Health, Labour and Welfare. Vital statistics 2001

2. Sasaki J, Arakawa K, Iwashita M, Matsushita Y, Kono S. Reduction in serum total cholesterol and risks of coronary events and cerebral infarction in Japanese men. Circ J 2003; 67: 473-478.

3. Iwashita M, Matsushita Y, Sasaki J, Arakawa K, Kono S. Relation of serum total cholesterol and other factors to risk of cerebral infarction in Japanese men with hypercholesterolemia. Circ J 2005; 69: 1-6.

4. Wolf PA, Kannel WB, Verter J. Current status of risk factors for stroke. Neurol Clin 1983; 1: 317-343.

5. Prospective Studies Collaboration. Cholesterol, diastolic blood pressure, and stroke: 13,000 strokes in 450,000 people in 45 prospective cohorts. Lancet 1995; 346: 1647-1653.

6. Nakayama T, Date C, Yokoyama T, Yoshiike N, Yamaguchi M, Tanaka H. A 15.5-year follow-up study of stroke in a Japanese provincial city: The Shibata study. Stroke 1997; 28: 45-52.

7. Tanaka H, Ueda Y, Hayashi M, Date C, Baba T, Yamashita H, et al. Risk factors for cerebral hemorrhage and cerebral infarction in a Japanese rural community. Stroke 1982; 13: 62-73.

8. Scandinavian Simvastatin Survival Study Group. Randomised trial of cholesterol lowering in 4444 patients with coronary heart disease: The Scandinavian Simvastatin Survival Study (4S). Lancet 1994; 344: $1383-1389$.

9. Byington RP, Jukema JW, Salonen JT, Pitt B, Bruschke AV, Hoen $\mathrm{H}$, et al. Reduction in cardiovascular events during pravastatin therapy: Pooled analysis of clinical events in the Pravastatin Atherosclerosis Intervention Program. Circulation 1995; 92: 2419-2425.

10. Heart Protection Study Collaborative Group. MRC/BHF Heart Pro- tection Study of cholesterol lowering with simvastatin in 20,536 high-risk individuals: A randomised placebo-controlled trial. Lancet 2002; 360: 7-22.

11. Sever PS, Dahlof B, Poulter NR, Wedel H, Beevers G, Caulfield M, et al. Prevention of coronary and stroke events with atorvastatin in hypertensive patients who have average or lower-than-average cholesterol concentrations, in the Anglo-Scandinavian Cardiac Outcomes Trial-Lipid Lowering Arm (ASCOT-LLA): A multicentre randomised controlled trial. Lancet 2003; 361: 1149-1158.

12. Soyama Y, Miura K, Morikawa Y, Nishijo M, Nakanishi Y, Naruse Y, et al. High-density lipoprotein cholesterol and risk of stroke in Japanese men and women: The Oyabe study. Stroke 2003; 34: $863-$ 868.

13. Eastern Stroke and Coronary Heart Disease Collaborative Research Group. Blood pressure, cholesterol, and stroke in eastern Asia. Lancet 1998; 352: $1801-1807$.

14. Matsuzaki M, Kita T, Mabuchi H, Matsuzawa Y, Nakaya, N, Oikawa $\mathrm{S}$, et al. Large scale cohort study of the relationship between serum cholesterol concentration and coronary events with low-dose simvastatin therapy in Japanese patients with hypercholesterolemia: Primary prevention cohort study of the Japan Lipid Intervention Trial (JLIT). Circ J 2002; 66: 1087-1095.

15. Mabuchi H, Kita T, Matsuzaki M, Matsuzawa Y, Nakaya N, Oikawa $\mathrm{S}$, et al. Large scale cohort study of the relationship between serum cholesterol concentration and coronary events with low-dose simvastatin therapy in Japanese patients with hypercholesterolemia and coronary heart disease: Secondary prevention cohort study of the Japan Lipid Intervention Trial (J-LIT). Circ J 2002; 66: 1096-1100.

16. Matsuzawa Y, Kita T, Mabuchi H, Matsuzaki M, Nakaya N, Oikawa $\mathrm{S}$, et al. Sustained reduction of serum cholesterol in low-dose 6-year simvastatin treatment with minimum side effects in 51,321 Japanese hypercholesterolemic patients: Implication of the J-LIT Study, a large scale nationwide cohort study. Circ J 2003; 67: 287-294.

17. Matsuzawa Y, Itakura H, Kita T, Mabuchi H, Matsuzaki M, Nakaya $\mathrm{N}$, et al. Design and baseline characteristics of a cohort study in Japanese patients with hypercholesterolemia: The Japan Lipid Intervention Trial (J-LIT). Curr Ther Res 2000; 61: 219-243.

18. Friedwald WT, Levy RI, Fredrickson DS. Estimation of the concentration of low-density lipoprotein cholesterol in plasma, without use of the preparative ultracentrifuge. Clin Chem 1972; 18: 499-502.

19. Okinaka S. Epidemiological study of stroke: Three-year follow-up study of 17 districts in Japan. Jpn Med $J$ 1966; 2221: 19-28 (in Japanese).

20. Corvol JC, Bouzamondo A, Sirol M, Hulot JS, Sanchez P, Lechat P. Differential effects of lipid-lowering therapies on stroke prevention. Arch Intern Med 2003; 163: 669-676.

21. Rosenson RS, Tangney CC. Antiatherothrombotic properties of statins: Implications for cardiovascular event reduction. JAMA 1998; 279: $1643-1650$. 